\title{
Hypoparathyroidism-replacing PTH
}

A recent double-blind, placebocontrolled randomized phase III trial has demonstrated that replacing parathyroid hormone (PTH) with the recombinant form PTH(1-84) is safe and effective.

Patients with hypoparathyroidism are currently treated with high doses of calcium and calcitriol (active vitamin D), which can cause serious adverse effects. "Hypoparathyroidism is one of the last classic endocrine disorders for which replacing the missing hormone is not an FDA-approved therapy," explains lead author Michael Mannstadt (Harvard Medical School, USA).

In this trial, 90 patients received PTH(1-84) and 44 received placebo for 24 weeks. The doses of calcium and calcitriol where progressively reduced, while PTH(1-84) was raised from $50 \mu \mathrm{g}$ to $75 \mu \mathrm{g}$ and then to $100 \mu \mathrm{g}$ up to week 5 .

The primary end point of reducing doses of calcium and calcitriol by at least $50 \%$ while maintaining serum levels of calcium within the target range was achieved in $53 \%$ of patients receiving $\mathrm{PTH}(1-84)$, compared with $2 \%$ of those given placebo. The number and severity of adverse events were similar for the two groups. These findings demonstrate that $\mathrm{PTH}(1-84)$ is effective, well-tolerated and safe in patients with hypoparathyroidism.

"Replacing PTH in patients with hypoparathyroidism is an exciting development, and the hope is that this treatment will be available as an option for patients with this disease," says Mannstadt. However, the researchers note that further work is needed to investigate the effects on renal and bone parameters.

Claire Greenhill

Original article Mannstadt, M. et al. Efficacy and safety of recombinant human parathyroid hormone (1-84) in hypoparathyroidism (REPLACE): a double-blind, placebocontrolled, randomised, phase 3 study. Lancet Diabetes Endocrinol. doi:10.1016/S2213-8587(13)70106-2 